\section{DIGITAL TRAVELLER IN CONNECTED VEHICLES}

Several technologies, communication networks and systems have been innovated and implemented to enable global vehicle connectivity for the digital traveller. Connectivity has become important in the context of mobility for reasons that could be commercial or personal, for product development, upgrade of systems' software or data management. The founding principle of connected vehicles is to be able to communicate with each other and with a remote station to enhance safety and efficiency, and reduce cost of mobility.

A study by the Texas Transportation Institute, quoted by IEA, estimated that in 2008 traffic congestion cost the US $\$ 87$ bn, 4.2 bn hours and 10.6 bn litres of oil. Connected mobility can reduce such traffic congestion by optimising traffic management. Worldwide, there are numerous programmes in ITS - Intellidrive in the US, Advanced Security Vehicle in Japan, INVENT and SimTD in Germany, PRETID in France, CGVS in UK, IVSS in Sweden, and in the EU - CVIS and SAFESPOT.

The digital traveller in connected vehicles sees several benefits and ease of comfort on the personal front - exchange personalised data and communicate seamlessly, real-time positioning, accident reporting and despatch of assistance, automatic vehicle speed control, being able to convey traffic patterns and alternate routes to vehicle, locate points of interest and driving directions, safety during weather conditions, and through telematics - ability to operate home, office or other security systems as necessity warrants.

From a product development perspective, connected vehicles and connectivity has opened many newer avenues to improve drivability, calibration of vehicles, remote diagnostics, fleet management of test data and upload of developmental software. The developmental time at the OEM end has reduced considerably and the quality of vehicles has improved. Data and activities that were only analysed at regular intervals after vehicles returned to workshop can now be monitored real time, with modifications being done for calibration as appropriate. Fleets of vehicles in different geographic zones and weather conditions are easily manageable now. Charging protocols and driving behaviour monitoring in XEV has helped enhance the growth of electric mobility infrastructure.

\section{COMMERCIAL VIABILITY}

The commercial advantages of connected vehicles are innumerable. Among others, efficiency improvement in cargo movement, delivery and maintenance schedules, traffic management, toll booth connectivity for revenue collection and emergency services are primary. Intelligent Transport Systems (ITS) also eases digital traveller movement for inter-city bus management and efficiency in parking. It is possible to monitor traffic density, help urban development, and highway and inner-city expansion strategies.

With the expansion of networks and communication protocols, the challenge is to be able to upgrade the security networks of connectivity and provide a secure data environment 'on the wheels'. Occupants working on encrypted data find it unsafe via connected vehicles,

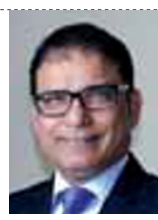

DR ARUN JAURA

Board of Directors, SAE International

though this is being mitigated by improving Near Field Communication. Perennially, occupants are seeking faster communication and more data transfer, which requires high-speed bandwidth. The challenge is to be able to provide vehicle connectivity with multiple devices and multiple occupants operating on different non-interchangeable networks from different providers. R\&D around networks, nano-chips and embedded software has grown exponentially to surpass these and other digital growing demands of clientele - public, private and government.

Interestingly, connected vehicles are the lego blocks for the 'Internet of Vehicle' it is prudent to continue development of V2X connectivity. V2X encompasses vehicle-to-vehicle, vehicle-to-sensors, vehicle-to-internet and vehicle-to-road infrastructure connectivity, along with aspects of data integration and telematics. The efficiency of overall connectivity is directly proportional to the number of vehicles and occupants on the roads.

Connected vehicles provide holistic efficient solutions facing 21 st century mobility, and this heralds a revolution for the 'digital traveller'.

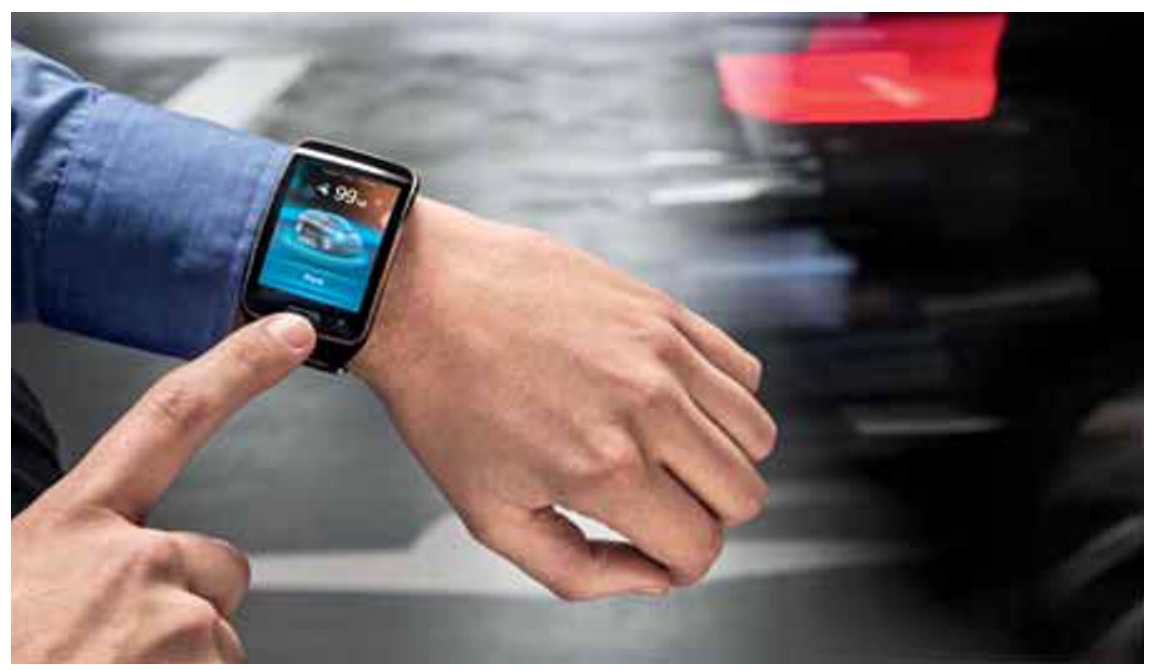

